\title{
Correlated Photon Emission from a Single II-VI Quantum Dot
}

\author{
C. Couteau \\ CEA-CNRS-UJF Joint Group Nanophysics and Semiconductors, \\ Laboratoire de Spectrométrie Physique, CNRS UMR 5588, \\ Université J. Fourier Grenoble 1, 38402 Saint-Martin d'Hères, France \\ S. Moehl, F. Tinjod, J.M. Gérard, K. Kheng, and H. Mariette \\ CEA-CNRS-UJF Joint Group Nanophysics and Semiconductors, \\ CEA/DRFMC/SP2M, 17 rue des Martyrs, 38054 Grenoble, France \\ J. A. Gaj \\ Institute of Experimental Physics, Warsaw University, Hoza 69, 00-681 Warsaw, Poland. \\ R. Romestain and J.P. Poizat \\ CEA-CNRS-UJF Joint Group Nanophysics and Semiconductors, \\ Laboratoire de Spectrométrie Physique, CNRS UMR 5588, \\ Université J. Fourier Grenoble 1, 38402 Saint-Martin d'Hères, France
}

\begin{abstract}
We report correlation and cross-correlation measurements of photons emitted under continuous wave excitation by a single II-VI quantum dot (QD) grown by molecular-beam epitaxy. A standard technique of microphotoluminescence combined with an ultrafast photon correlation set-up allowed us to see an antibunching effect on photons emitted by excitons recombining in a single $\mathrm{CdTe} / \mathrm{ZnTe}$ $\mathrm{QD}$, as well as cross-correlation within the biexciton $\left(X_{2}\right)$-exciton $(X)$ radiative cascade from the same dot. Fast microchannel plate photomultipliers and a time-correlated single photon module gave us an overall temporal resolution of 140 ps better than the typical exciton lifetime in II-VI QDs of about 250ps.

PACS numbers:
\end{abstract}

The generation of triggered single photons has brought a lot of interest in the last few years owing to its application in quantum cryptography [1, 2] and its possible use for quantum computation [3]. Recent experiments have shown that self-assembled quantum dots (SAQDs) are potentially a good candidate for the production of triggered single photons [2, 4, 5, 6]. In order to implement Knill et al.'s proposal [3], one of the very stringent requirement is to produce indistinguishable single photons to enable multi-photon interference effects [7]. The possibility of creating on demand a single polarization entangled photon pair directly from a SAQD has also been proposed [8]. In this case, the production of the entangled pair of photons comes from the recombination of two excitons within the same QD $[\underline{9}]$. Polarization correlation measurements of the biexcitonic $\left(X_{2}\right)$ cascade to the ground state via the exciton $\left(X_{1}\right)$ state have been reported recently in III-V [10] and II-VI [11] compounds. However, no entanglement has been seen so far in the $X_{2}$ cascade.

Most of the results on single photon sources using SAQDs have been obtained with III-V semiconductors. Only a few recent papers [6, 11, 12] present photon correlation measurements on II-VI SAQDs, all with CdSe/ZnSe. The family of II-VI materials exhibits interesting features for quantum information applications. The lifetime (of the order of $200 \mathrm{ps}$ ) is smaller than in III-V SAQDs. It is an advantage for the production of indistinguishable single photons since this means that the emission of a photon takes less time and should then be less sensitive to dephasing. This short lifetime could also allow higher repetition rate (in the $\mathrm{GHz}$ range).

In this letter, we report the observation of antibunching of the excitonic line of $\mathrm{CdTe} / \mathrm{ZnTe} \mathrm{SAQD}$, and the biexciton-exciton cross-correlation under continuous wave $(\mathrm{cw})$ excitation. The short lifetimes in II-VI semiconductors have made it necessary to set-up an ultrafast photon correlation experiment using rapid microchannel plate photomultipliers.

The investigated sample consists of a CdTe SAQD layer (with dot density of about $310^{10} \mathrm{~cm}^{-2}$ ) embedded in ZnTe barriers. A detailed description of the sample growth can be found in 13. Single dot spectroscopy is obtained by exciting the sample through submicron apertures (with sizes down to $0.2 \mu \mathrm{m}$ ) made in a thin (100nm) aluminium mask deposited onto the surface. The sample is mounted on the cold finger of a helium flux cryostat keeping the QDs at about $5 \mathrm{~K}$ for all the experiments described here. A microscope objective (numerical aperture of 0.4$)$ and a high refractive index $(n=2.16)$ hemispherical solid immersion lens (SIL) 14] are used to collect the single dot emission efficiently. The $488 \mathrm{~nm}(2.541 \mathrm{eV})$ line of a cw argon laser is sent through the same optics for the excitation. Fine positioning of the laser onto the chosen aperture in the mask is obtained with submicronic displacements of the microscope objective by XYZ piezo drivers.

The collected QD photoluminescence (PL) is then 


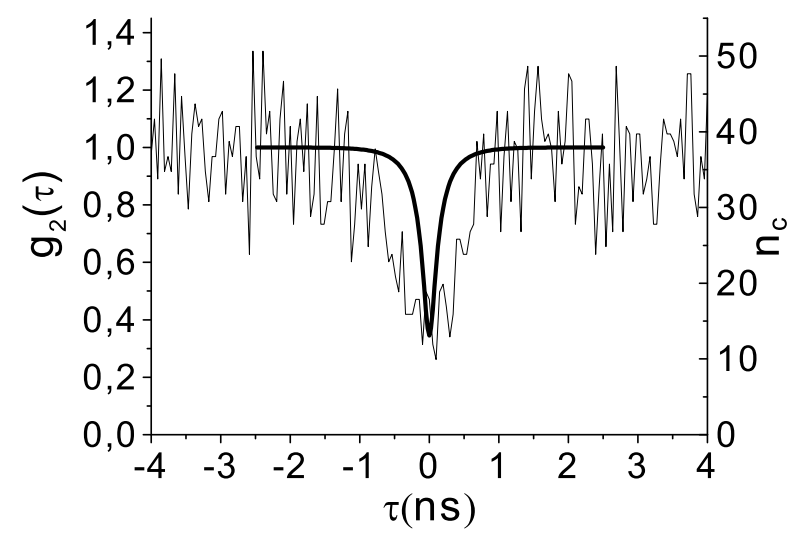

FIG. 1: Autocorrelation measurement of a single exciton. The left axis is the autocorrelation function $g^{(2)}(\tau)$ and the right axis is the unormalized coincidence rate. The time bin is $49 \mathrm{ps}$ and $n_{c}$ is the total number of events during the 12600s of acquisition. Typical counting rates were $n_{\text {start }}=10000 \mathrm{cps}$ and $n_{\text {stop }}=7000 \mathrm{cps}$ on a single exciton line. The background count rates of each channel were $n_{\text {start }}^{d}=1000 \mathrm{cps}$ and $n_{\text {stop }}^{d}=800 \mathrm{cps}$. The boldline is the expected curve for a multiexcitonic model, see text.

sent on a 50/50 beamsplitter for correlation measurements 15. In each arm of the beamsplitter, the light is dispersed by a monochromator (grating of 1200 grooves $/ \mathrm{mm}, 30 \mathrm{~cm}$ and $50 \mathrm{~cm}$ focal length respectively). Each monochromator has a switchable mirror inside that can direct the luminescence either onto a CCD camera for the measurement of the PL spectrum or through the exit slit towards the single photon counter. In our case these detectors are microchannel plate photomultipliers that have a very small transit time jitter $(<50 \mathrm{ps})$. The detectors send electrical pulses into a time-correlated single photon module that contains all the electronics necessary for the data processing. The overall temporal resolution of our set-up is essentially limited by the jitter of the detectors and the dispersion of the monochromator gratings. This time resolution was measured by recording the autocorrelation function of $200 \mathrm{fs}$ long pulses from a frequency-doubled Ti:Sapphire laser at $80 \mathrm{MHz}$ repetition rate. A FWHM of 140ps was obtained for the autocorrelation function peaks. This is, to our knowledge, the shortest time resolution ever reported for photon correlation measurements.

Fig [1 shows the autocorrelation function $g^{(2)}(\tau)$ of a $\mathrm{QD}$ excitonic line at $2.232 \mathrm{eV}(555.5 \mathrm{~nm})$. It displays the reduced coincidence rate around $\tau=0$ (antibunching), which is characteristic of the photon statistics of a single emitter. We have chosen to study excitonic lines on the high energy side of the CdTe QDs emission distribution because the sensitivity of our detectors drops very quickly at lower energies. In these measurements, we have performed a careful calibration of the pump power in unit of $\Gamma_{X_{1}}=1 / T_{X_{1}}$ where $T_{X_{1}}$ is the lifetime of the

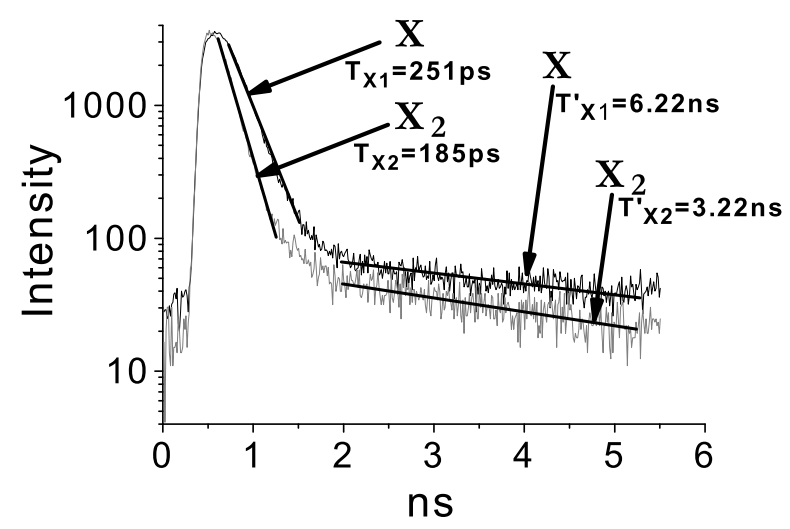

FIG. 2: Lifetime measurements of the exciton and its associated biexciton done with a pulsed Ti:Sapphire laser.

exciton. This has been done by fitting the luminescence intensity versus the pump power using a multiexciton ladder model [16] with a population of up to 4 excitons in the dot. In this model the lifetimes of the first two excitons $\left(X_{1}\right.$ and $\left.X_{2}\right)$ were taken from experimental measurements (see below), and the lifetimes of the triexciton $\left(X_{3}\right)$ and the quadriexciton $\left(X_{4}\right)$, which are not very critical, were taken from ref [17]. The autocorrelation function of Fig 1 was obtained with a QD excitation rate $r=0.65 \Gamma_{X_{1}}$.

After convolution with the time resolution of our setup, and taking into account the signal to background ratio [18], the bold line in Fig 1 is the prediction of the ladder model for the autocorrelation function. There is a difference of a factor of 4 between the FWHM of the experimental curve and the model prediction. This mismatch has been observed on the three quantum dots of the same sample that we have studied. Previous groups [19, 20] have observed the same mismatch in the past for III-V compounds. The presence of dark excitons may explain this discrepancy as suggested by the measurements described in the following.

Decay time measurements (see Fig 21) using a pulsed Ti:Sapphire laser (pulse duration 1ps) give lifetime values of $T_{X_{1}}=251 \pm 5 \mathrm{ps}$ and $T_{X_{2}}=185 \pm 4 \mathrm{ps}$ for the exciton and its associated biexciton respectively. A biexponential decay is observed both for $X_{1}$ and $X_{2}$, with $T_{X_{1}}^{\prime}=6.22 \pm 0.68 \mathrm{~ns}$ and $T_{X_{2}}^{\prime}=3.22 \pm 0.16 \mathrm{~ns}$ for their slow components. This longer time decay might be the signature of the presence of the dark exciton as seen in 21]. Nevertheless, further investigations, like temperature dependent measurements, need to be done to resolve the question.

Fig 3 shows the correlation measurement between the same exciton and its biexciton [9, 16]. For that, we used the same set-up, but one of the spectrometers was tuned on the wavelength of the biexcitonic line at $2.219 \mathrm{eV}$ (558.6nm), that is $13 \mathrm{meV}$ below the single exciton emis- 


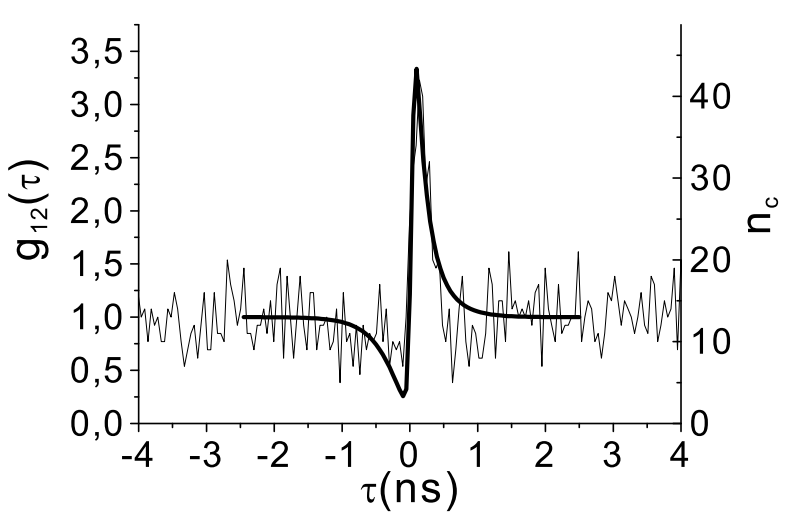

FIG. 3: Cross-correlation measurement between an exciton and its biexciton. The bold line is the expected curve for the same multiexcitonic model, see text for details. $n_{c}$ is again the total number of events during the 9 720s of acquisition. The time bin is 49ps. Counting rates are $n_{2}=4800 \mathrm{cps}$ for the $X_{2}$ and $n_{1}=7600 \mathrm{cps}$ for the $X_{1}$ with background count rates of $n_{2}^{d}=400 \mathrm{cps}$ and $n_{2}^{d}=600$ cps.

sion. The pump parameter was $r=0.204 \Gamma_{X_{1}}$. When $X_{2}$ triggers the start and $X_{1}$ stops the time counter, a discontinuity at $\tau=0$ is expected with value above unity for $\tau>0$ revealing the bunching part of the cascade 9 . For $\tau<0$, antibunching is expected, since a recycling time is necessary for a biexciton photon to be emitted after the last exciton has been recombined.

Our result in Fig 3 displays very clearly the bunching part for positive times, and less clearly the antibunching part for negative times. The bold line is the fitting curve using the same ladder model with up to 4 excitons in the dot. Now, we observe that, contrary to the antibunching measurement in Fig 1 we do have the correct decay time corresponding to $T_{X_{1}}$ for positive times. The discrepancy of a factor of 4 for the exciton autocorrelation is no longer present in cross-correlation measurements. This indeed suggests an influence of dark excitons which would explain the discrepancy of Fig 1 since in crosscorrelation experiments the recombination of a biexciton always leaves an exciton in a bright state.

To conclude, thanks to our temporal resolution, we presented in this letter cw autocorrelation and crosscorrelation measurements of a single self-assembled quantum dot made of II-VI materials. We reported expected and unexpected behaviors from these results as discussed above. Further experiments using pulsed excitation need doing in $\mathrm{CdTe} / \mathrm{ZnTe} \mathrm{QDs}$ but the first results shown here present a good omen that such heterostructures might be good candidates for the purposes of quantum optics and quantum information.

We thank Ph. Grangier for the loan of the two microchannel plate photomultipliers, M. Terrier for the processing of the sample, and F. Donatini for experimental assistance. This work is supported by the French Ministry for Research (ACI "Polqua", and ACI Jeune Chercheur) and the "Sciences et Technologies de l'Information et de la Communication" Department of the CNRS. J.A.G. acknowledges the support of Polish Committee for Scientific Research (grant No PBZ-KBN044/P03/2001).

We would like to dedicate this paper to the memory of Robert Romestain who died in a mountain accident during the writting of this paper.
[1] A. Beveratos, R. Brouri, T. Gacoin, A. Villing, J.Ph. Poizat, and Ph. Grangier, Phys. Rev. Lett. 89, 187901 (2002)

[2] E. Waks, K. Inoue, C. Santori, D. Fattal, J. Vuckovic, G.S. Solomon, and Y. Yamamoto,Nature (London) 420, 762 (2002).

[3] E. Knill, R. Laflamme, and G.J. Milburn, Nature (London) 409, 46 (2001).

[4] J.M. Gérard and B. Gayral, J. Lightwave Technol. 17, 2089 (1999).

[5] P. Michler, A. Kiraz, C. Becher, W.V. Schoenfeld, P.M. Petroff, L. Zhang, E. Hu, and A. Imamoglu, Science 290, 2282 (2000).

[6] K. Sebald, P. Michler, T. Passow, D. Hommel, G. Bacher, and A. Forchel, Apll. Phys. Lett. 81, 2920 (2002).

[7] C. Santori, D. Fattal, J. Vuckovic, G. S. Solomon, and Y. Yamamoto, Nature (London) 419, 594 (2002).

[8] O. Benson, C. Santori, M. Pelton, and Y. Yamamoto, Phys. Rev. Lett. 84, 2513 (2000).

[9] E. Moreau, I. Robert, L. Manin, V. Thierry-Mieg, J.M. Gérard, and I. Abram, Phys. Rev. Lett. 87, 183601 (2001).
[10] C. Santori, D. Fattal, M. Pelton, G. S. Solomon, and Y. Yamamoto, Phys. Rev. B 66, 45308 (2002).

[11] S.M. Ulrich, S. Strauf, P. Michler, G. Bacher, and A. Forchel, App. Phys. Lett. 83, 1848 (2003).

[12] T. Aichele, V. Zwiller, O. Benson, I. Akimov, and F. Henneberger, J. Opt. Soc. Am B 20, 2189 (2003).

[13] F. Tinjod, B. Gilles, S. Moehl, K. Kheng, and H. Mariette, App. Phys. Lett. 82, 4340 (2003).

[14] S. Moehl, Hui Zhao, B. Dal Don, S. Wachter, and H. Kalt, J. App. Lett. 93, 6265 (2002); W.L. Barnes, G. Björk, J.M. Gérard, P. Jonsson, J.A.E Wasey, P.T. Worthing, and V. Zwiller, Eur. Phys. J. D 18, 197 (2002).

[15] R. Hanbury Brown, and R. Q. Twiss, Nature (London) 177, 27, (1956).

[16] D.V. Regelman, U. Mizrahi, D. Gershoni, E. Ehrenfreund, W.V. Schoenfeld, and P.M. Petroff, Phys. Rev. Lett. 87, 257401 (2001).

[17] L. Besombes, Thèse de Doctorat, Université Joseph Fourier Grenoble (2002).

[18] R. Brouri, A. Beveratos, J.Ph. Poizat, and Ph. Grangier, Opt. Lett. 25, 1294 (2000).

[19] C. Becher, A. Kiraz, P. Michler, A. Imamoglu, W.V. 
Schoenfeld, P.M. Petroff, L. Zhang, and E. Hu, Phys. Rev. B 63, 121312 (2001).

[20] E. Moreau, Thèse de Doctorat, Université Paris-Sud Orsay (2002).
[21] O. Labeau, P. Tamarat, and B. Lounis, Phys. Rev. Lett. 90, 257404 (2003). 